\title{
(2) OPEN ACCESS \\ Development and validation of a deep learning system to classify aetiology and predict anatomical outcomes of macular hole
}

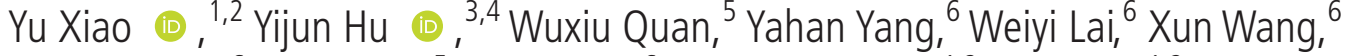 \\ Xiayin Zhang, ${ }^{6}$ Bin Zhang, ${ }^{5}$ Yuqing Wu ${ }^{6}$ Qiaowei Wu (1) ${ }^{1,2}$ Baoyi Liu, ${ }^{1,2}$ \\ Xiaomin Zeng, ${ }^{1,2}$ Zhanjie Lin, ${ }^{1}$ Ying Fang, ${ }^{1}$ Yu Hu, ${ }^{7}$ Songfu Feng, ${ }^{8}$ Ling Yuan, ${ }^{7}$ \\ Hongmin Cai, ${ }^{5}$ Tao Li (1), ${ }^{6}$ Haotian Lin (1) , ${ }^{6,9}$ Honghua Yu (1) ${ }^{1,2}$
}

\begin{abstract}
- Additional supplemental material is published online only. To view, please visit the journal online (http://dx.doi. org/10.1136/bjophthalmol2021-318844).
\end{abstract}

For numbered affiliations see end of article.

\section{Correspondence to}

Professor Haotian Lin and Professor Tao Li, State Key Laboratory of Ophthalmology, Zhongshan Ophthalmic Center, Sun Yat-sen University, Guangzhou, China; gddlht@aliyun.com lita02@mail.sysu.edu.cn and Professor Honghua Yu. Guangdong Eye Institute, Department of Ophthalmology, Guangdong Provincial People's Hospital, Guangdong Academy of Medical Sciences, Guangzhou, China; yuhonghua@gdph.org.cn

YX, YH and WQ contributed equally.

Received 11 January 2021 Accepted 23 July 2021

\begin{abstract}
Aims To develop a deep learning (DL) model for automatic classification of macular hole ( $\mathrm{MH}$ ) aetiology (idiopathic or secondary), and a multimodal deep fusion network (MDFN) model for reliable prediction of $\mathrm{MH}$ status (closed or open) at 1 month after vitrectomy and internal limiting membrane peeling (VILMP).
\end{abstract}

Methods In this multicentre retrospective cohort study, a total of $330 \mathrm{MH}$ eyes with 1082 optical coherence tomography (OCT) images and 3300 clinical data enrolled from four ophthalmic centres were used to train, validate and externally test the DL and MDFN models. 266 eyes from three centres were randomly split by eye-level into a training set (80\%) and a validation set $(20 \%)$. In the external testing dataset, 64 eyes were included from the remaining centre. All eyes underwent macular OCT scanning at baseline and 1 month after VILMP. The area under the receiver operated characteristic curve (AUC), accuracy, specificity and sensitivity were used to evaluate the performance of the models.

Results In the external testing set, the AUC, accuracy, specificity and sensitivity of the $\mathrm{MH}$ aetiology classification model were $0.965,0.950,0.870$ and 0.938 , respectively; the AUC, accuracy, specificity and sensitivity of the postoperative $\mathrm{MH}$ status prediction model were $0.904,0.825,0.977$ and 0.766 , respectively; the AUC, accuracy, specificity and sensitivity of the postoperative idiopathic $\mathrm{MH}$ status prediction model were $0.947,0.875,0.815$ and 0.979 , respectively. Conclusion Our DL-based models can accurately classify the $\mathrm{MH}$ aetiology and predict the $\mathrm{MH}$ status after VILMP. These models would help ophthalmologists in diagnosis and surgical planning of $\mathrm{MH}$.

\section{INTRODUCTION}

Macular hole $(\mathrm{MH})$, a full-thickness defect of the neurosensory retina tissue at the fovea, is one of the reasons for central vision deterioration. ${ }^{1} \mathrm{MH}$ can be divided into two categories according to the aetiology. The aetiology of idiopathic $\mathrm{MH}$ (IMH) is unknown, while secondary $\mathrm{MH}(\mathrm{SMH})$ is caused by known aetiologies like high myopia and trauma. $\mathrm{IMH}$ is the most common type of $\mathrm{MH}$. It is estimated to affect $0.1 \%-0.8 \%$ of the population aged over 44 years, and nearly two-thirds of patients with IMH are female. ${ }^{2-4}$ IMH development is considered as pathological vitreoretinal traction at the central macula. ${ }^{5}$ Patients with IMH with progressive visual impairment and metamorphopsia usually require surgical intervention. ${ }^{6}$ Vitrectomy and internal limiting membrane peeling (VILMP) has been proved effective to treat full-thickness IMH with success rates of $80 \%-95 \% .{ }^{78}$ However, IMH remains open after routine VILMP in some cases. ${ }^{9}$ For instance, it was reported that up to $44 \%$ of large MHs remained open after first surgery. ${ }^{10}$ In patients with an open IMH after initial surgery, a second surgery is often mandatory. ${ }^{11}$ However, second surgery is typically associated with higher medical costs and less promising visual outcomes. $\mathrm{SMH}$ is usually caused by high myopia and blunt ocular trauma. ${ }^{12} 13$ The pathogenesis of SMH is more complicated and the success rate of initial repair is lower than that of IMH. ${ }^{12}{ }^{14}$ Therefore, it is important to distinguish IMH and $\mathrm{SMH}$, and to predict the postoperative $\mathrm{MH}$ status after initial repair during surgical planning.

Optical coherence tomography (OCT) has been widely used in diagnosis and prognosis assessment of MH. ${ }^{13}{ }^{15} \mathrm{IMH}$ may have different OCT images from $\mathrm{SMH}$, especially those secondary to high myopia, with its own OCT characteristics. ${ }^{12}$ For prognosis assessment, some OCT parameters have been identified as factors related to outcomes of $\mathrm{MH}$ surgeries. These factors include the minimum diameter of $\mathrm{MH}(\mathrm{MIN})$, the base diameter of $\mathrm{MH}$ (BASE), hole form factor (HFF), macular hole index (MHI), tractional hole index (THI) and diameter hole index (DHI). ${ }^{121316-19}$ However, many previous studies mainly focused on the prediction abilities of one OCT parameter only. ${ }^{15}$

Deep learning (DL) is an emerging artificial intelligence technology, which has been successfully applied in various areas, such as image identification and classification. ${ }^{20} 21$ Convolutional neural network (CNN), one of the most popular DL models, employs multiple convolutional layers for automatic identification and extraction of feature representations. A CNN-based DL model was recently developed to automatically detect IMH using ultra-wide-field fundus images. ${ }^{22}$ In addition to the CNN, the multimodal deep fusion network (MDFN) is an important technology to exploit the comprehensive information of multimodal data 
extracted by different unimodal DL models. Different feature sets of the multimodal data can be fused by MDFN to improve the performance of the DL model. ${ }^{23}$ Given the fact that the surgical outcome of $\mathrm{MH}$ is affected by both morphological factors (such as preoperative macular OCT parameters) and other clinical factors (such as duration of $\mathrm{MH}$ ), MDFN is potentially helpful in the prediction of postoperative $\mathrm{MH}$ status by combining the information of these factors.

The current study aimed to develop a DL model for automatic classification of $\mathrm{MH}$ aetiology based on macular OCT images, and an MDFN-based DL model to automatically predict $\mathrm{MH}$ status after VILMP surgery using preoperative OCT images and other clinical data. We also compared the performance of the MDFN model with that of the unimodal models.

\section{METHODS}

\section{Participants and data collection}

In this multicentre retrospective cohort study, eyes with fullthickness $\mathrm{MH}$ (ie, IMH and $\mathrm{SMH}$ caused by high myopia or trauma) followed up for at least 1 month after VILMP surgery were included. The surgical process of VILMP is described in the online supplemental eMethod 1. The patients' age, gender and duration of symptoms (eg, progressive visual impairment and metamorphopsia) were extracted from the electronic medical records (EMR). All of the eyes underwent ophthalmologic examinations including slit-lamp biomicroscope anterior segment and fundus examination, and spectral domain-OCT scanning (SD-OCT, Spectralis; Heidelberg Engineering, Heidelberg, Germany) at baseline and 1 month after VILMP. Additional details on OCT examination and OCT parameter measurements are presented in the online supplemental eMethod 2.

$\mathrm{MH}$ eyes were recruited retrospectively between January 2014 and August 2020. Among the $349 \mathrm{MH}$ eyes of 334 patients with 1125 preoperative macular OCT images received from four ophthalmic settings, 5 eyes were excluded due to missing preoperative OCT images, 8 eyes were excluded due to missing postoperative OCT images and 6 eyes were excluded due to insufficient quality of OCT images. The remaining 330 eyes of 315 patients with 1082 preoperative macular OCT images (285 IMH eyes with 957 images and $45 \mathrm{SMH}$ eyes with 125 images (36 eyes with 106 images secondary to high myopia, 9 eyes with 19 images secondary to trauma)) and 3300 clinical data (preoperative macular OCT parameters extracted from the OCT device and clinical data from EMR) were used to train, validate and externally test the $\mathrm{MH}$ aetiology classification model and postoperative $\mathrm{MH} / \mathrm{IMH}$ status prediction model. Of these eyes, $266 \mathrm{MH}$ eyes (232 IMH eyes and $34 \mathrm{SMH}$ eyes) collected from the Zhongshan Ophthalmic Center, the Department of Ophthalmology in the Zhujiang Hospital of Southern Medical University and the Department of Ophthalmology in the First Affiliated Hospital of Kunming Medical University were randomly divided into the training set ( $80 \%$ of the eyes) and the validation set (the other 20\% of the eyes). A separate set of $64 \mathrm{MH}$ eyes (53 IMH eyes and $11 \mathrm{SMH}$ eyes) collected from the Department of Ophthalmology in the Guangdong Provincial People's Hospital was used for external testing. The flow chart of the research performed in this study is shown in figure 1 .

\section{Development of DL model for MH aetiology classification}

The Visual Geometry Group network (VGG, Department of Engineering Science, The University of Oxford, Oxford, UK) with 16 convolutional layers was employed for the classification of $\mathrm{MH}$ aetiology. ${ }^{24}$ Preoperative macular OCT images were used

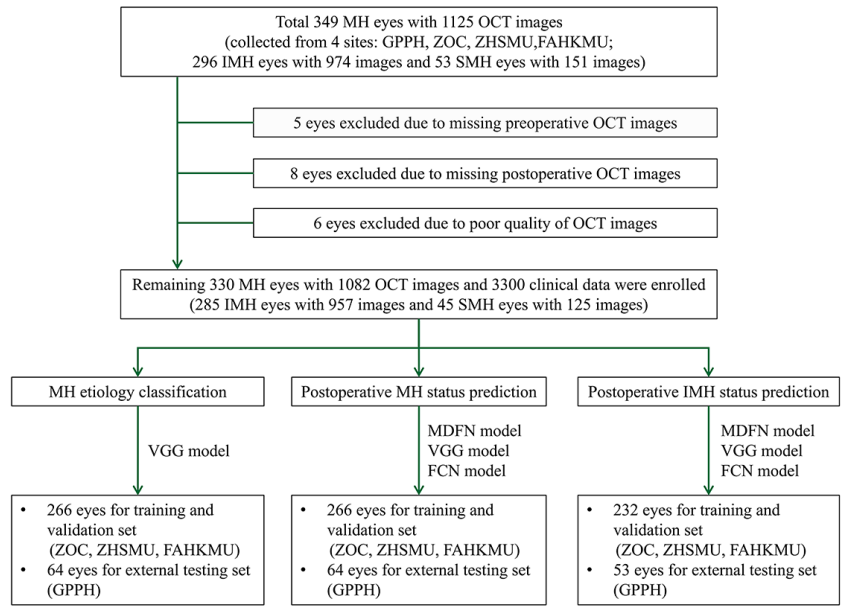

Figure 1 Flow chart of the research performed in this study. FAHKMU, First Affiliated Hospital of Kunming Medical University; FCN, fully connected network; GPPH, Guangdong Provincial People's Hospital; IMH, idiopathic macular hole; MDFN, multimodal deep fusion network; $\mathrm{MH}$, macular hole; $\mathrm{OCT}$, optical coherence tomography; SMH, secondary macular hole; VGG, Visual Geometry Group; ZHSMU, Zhujiang Hospital of Southern Medical University; ZOC, Zhongshan Ophthalmic Center.

as the input data. Based on the requirements of VGG network, the OCT images were preprocessed to normalise the input data. We removed saturated pixels from the raw OCT images with an intensity value of 255 , followed by resizing them into $224 \times 224$ pixels. A threshold probability value of 0.5 was used to classify the $\mathrm{MH}$ aetiology (ie, $\mathrm{SMH}$ if the value was 0.5 or more, or IMH if the value was <0.5) (figure 2). A detailed description of $\mathrm{MH}$ aetiology labelling and training procedure is described in the online supplemental eMethod 3.

\section{Development of DL models for MH/IMH status prediction}

Considering that the pathogenesis of $\mathrm{SMH}$ is more complicated and the success rate of initial repair is even lower than IMH, two prediction tasks were performed. For the first prediction task, all of the $330 \mathrm{MH}$ eyes, including both $45 \mathrm{SMH}$ eyes and $285 \mathrm{IMH}$ eyes, were included to predict the postoperative $\mathrm{MH}$ status. For the second prediction task, only 285 IMH eyes were enrolled to predict the postoperative status of IMH.

Preoperative macular OCT images and clinical data (ie, age, gender, duration of symptoms, MIN, BASE, height of hole, MHI, HFF, DHI and THI) of MH/IMH eyes were used as the input data. The DL model used for $\mathrm{MH}$ aetiology classification (VGG network) was also applied to obtain deep features of OCT images for $\mathrm{MH} / \mathrm{IMH}$ status prediction. In addition, the fully connected (FC) network was employed to obtain deep features of the clinical data.

After the two types of feature sets (multimodal deep features) were extracted, the VGG network, with the top output layer removed, was connected to the FC network. Moreover, the multimodal deep features were fused to have a comprehensive characterisation of $\mathrm{MH} / \mathrm{IMH}$. The concatenate operation could integrate semantic data obtained from diverse feature maps, thereby increasing the number of channels and improving model performance. The fused features were linked to a two-channel softmax layer via the FC layer, to arrive at the predictive probability value of $\mathrm{MH} / \mathrm{IMH}$ status. A threshold probability value of 0.5 was used to predict $\mathrm{MH} / \mathrm{IMH}$ status (ie, closed if the value 


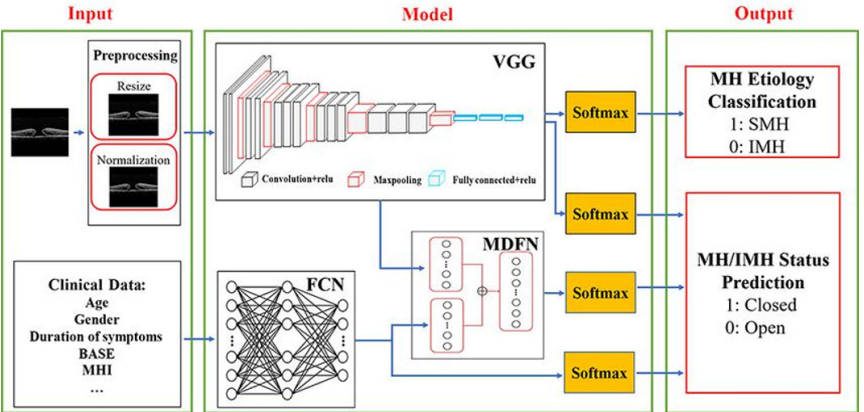

Figure 2 Demonstration of construction of deep learning models. BASE, base diameter of MH; FCN, fully connected network; IMH, idiopathic macular hole; MDFN, multimodal deep fusion network; $\mathrm{MHI}$, macular hole index; $\mathrm{MH}$, macular hole; $\mathrm{SMH}$, secondary macular hole; VGG, Visual Geometry Group. The deep learning models were trained to classify the $\mathrm{MH}$ aetiology (ie, SMH or IMH), and to predict postoperative $\mathrm{MH} / \mathrm{IMH}$ status (ie, closed or open) following initial repair.

was 0.5 or more, or open if the value was $<0.5$ ). A detailed description of postoperative $\mathrm{MH}$ status labelling and training procedure is given in the online supplemental eMethod 4.

To compare the predictive performance of the MDFN model to those of the unimodal DL models, we divided the MDFN model into two unimodal DL models with the same structure of VGG network and FC network. We first used the VGG network and the FC network to extract deep features of OCT images and clinical data, respectively. Then the $\mathrm{MH} / \mathrm{IMH}$ status was predicted by the unimodal DL models via the softmax layer. Schematic illustration of the DL models is presented in figure 2.
Statistical analysis is demonstrated in the online supplemental eMethod 5. In addition, we generated heatmaps to aid interpretation of the results and increase model transparency (described in the online supplemental eMethod 6).

\section{RESULTS}

Demographic data of the eyes enrolled in this study are summarised in table 1 . All patients were of the same ethnicity (Chinese). Among the total $330 \mathrm{MH}$ eyes, 217 eyes were from female patients (66\%), and the mean age of patients was $59.54 \pm 11.03$ years. There were 229 eyes with a closed $\mathrm{MH}$ (209 IMH and $20 \mathrm{SMH}$ ) and 101 eyes with an open $\mathrm{MH}$ (76 $\mathrm{IMH}$ and $25 \mathrm{SMH}$ ) at the 1 -month visit.

The performance metrics and the ROC curves of DL models are shown in table 2 and figure 3, respectively. The confusion matrices for external testing set are presented in the online supplemental eResult 1. For $\mathrm{MH}$ aetiology classification, the AUC of the DL model in the training set was 1.000, with an accuracy of 0.998 , specificity of 1.000 and sensitivity of 1.000 . In the validation set, the DL model showed an AUC of 0.997 , with an accuracy of 0.986 , specificity of 0.994 and sensitivity of 0.964. In the external testing set, the AUC, accuracy, specificity and sensitivity were $0.965,0.950,0.870$ and 0.938 , respectively (table $2 \mathrm{~A}$ and figure $3 \mathrm{~A}$ ).

For postoperative $\mathrm{MH}$ status prediction, the AUC of the MDFN model in the training set was 0.928 , with an accuracy of 0.855 , specificity of 0.897 and sensitivity of 0.808 . In the validation set, the AUC, accuracy, specificity and sensitivity of the MDFN model were $0.881,0.826,0.746$ and 0.912 , respectively. For external testing, the MDFN model achieved an AUC

Table 1 Baseline characteristics of the eyes*

\begin{tabular}{|c|c|c|c|c|}
\hline A. Baseline characteristics of MH eyes* & $\begin{array}{l}\text { Training set } \\
(n=199)\end{array}$ & $\begin{array}{l}\text { Validation set } \\
(n=67)\end{array}$ & $\begin{array}{l}\text { Testing set } \\
(n=64)\end{array}$ & $\begin{array}{l}\text { All eyes } \\
(n=330)\end{array}$ \\
\hline Aetiology, IMH, n (\%) & $173(86.93 \%)$ & $59(88.06 \%)$ & $53(82.81 \%)$ & $285(86.36 \%)$ \\
\hline Gender, female, n (\%) & $133(66.83 \%)$ & $43(64.18 \%)$ & $41(64.06 \%)$ & $217(65.76 \%)$ \\
\hline Age, years & $59.30(10.76)$ & $58.24(11.76)$ & $61.66(10.74)$ & $59.54(11.03)$ \\
\hline Duration of symptoms, months & $7.28(11.38)$ & $9.60(22.88)$ & $7.67(16.55)$ & $7.83(15.44)$ \\
\hline $\mathrm{MIN}, \mu \mathrm{m}$ & $437.39(204.75)$ & $484.88(192.94)$ & $436.05(238.46)$ & $446.77(210.32)$ \\
\hline BASE, $\mu \mathrm{m}$ & $955.78(460.20)$ & $1004.75(343.57)$ & $977.34(429.57)$ & $969.91(433.41)$ \\
\hline Height, $\mu \mathrm{m}$ & $439.37(91.80)$ & $464.66(102.91)$ & $467.69(124.10)$ & 449.99 (101.94) \\
\hline THI & $1.50(2.44)$ & $1.24(1.03)$ & $2.07(2.42)$ & $1.56(2.24)$ \\
\hline $\mathrm{DHI}$ & $0.48(0.16)$ & $0.49(0.13)$ & $0.45(0.18)$ & $0.48(0.16)$ \\
\hline $\mathrm{MHI}$ & $0.59(0.43)$ & $0.57(0.50)$ & $0.68(0.70)$ & $0.60(0.51)$ \\
\hline HFF & $0.77(0.22)$ & $0.76(0.16)$ & $0.88(0.36)$ & $0.79(0.25)$ \\
\hline B. Baseline characteristics of IMH eyes* & $\begin{array}{l}\text { Training set } \\
(n=174)\end{array}$ & $\begin{array}{l}\text { Validation set } \\
(n=58)\end{array}$ & $\begin{array}{l}\text { Testing set } \\
(n=53)\end{array}$ & $\begin{array}{l}\text { All eyes } \\
(n=285)\end{array}$ \\
\hline Gender, female, n (\%) & $113(64.94 \%)$ & $38(65.52 \%)$ & $35(66.04 \%)$ & $186(65.26 \%)$ \\
\hline Age, years & $59.36(11.27)$ & $60.67(9.33)$ & $63.77(8.96)$ & $60.45(10.63)$ \\
\hline Duration of symptoms, months & $7.18(11.60)$ & $6.77(11.04)$ & $7.35(16.53)$ & $7.13(12.56)$ \\
\hline $\mathrm{MIN}, \mu \mathrm{m}$ & $453.85(198.63)$ & $434.71(214.21)$ & $413.11(238.00)$ & $442.38(210.27)$ \\
\hline $\mathrm{BASE}_{,} \mu \mathrm{m}$ & $952.63(323.98)$ & $884.66(405.20)$ & 898.34 (391.39) & $928.70(356.23)$ \\
\hline Height, $\mu \mathrm{m}$ & $447.36(85.82)$ & $439.97(89.87)$ & $467.17(116.15)$ & $449.54(93.43)$ \\
\hline THI & $1.45(2.56)$ & $1.44(1.16)$ & $2.23(2.55)$ & $1.59(2.36)$ \\
\hline $\mathrm{DHI}$ & $0.48(0.15)$ & $0.52(0.15)$ & $0.46(0.19)$ & $0.48(0.16)$ \\
\hline $\mathrm{MHI}$ & $0.53(0.25)$ & $0.72(0.65)$ & $0.75(0.75)$ & $0.61(0.49)$ \\
\hline HFF & $0.75(0.17)$ & $0.81(0.28)$ & $0.90(0.38)$ & $0.79(0.25)$ \\
\hline
\end{tabular}

*Presented with mean (SD) except for aetiology and gender.

BASE, base diameter of MH; DHI, diameter hole index; HFF, hole form factor; IMH, idiopathic macular hole; MH, macular hole; MHI, macular hole index; MIN, minimal diameter of $\mathrm{MH} ; \mathrm{THI}$, tractional hole index. 


\begin{tabular}{|c|c|c|c|c|}
\hline $\begin{array}{l}\text { A. Performance of the DL } \\
\text { model for MH aetiology } \\
\text { classification }\end{array}$ & AUC & $\mathrm{ACC}$ & SPE & SEN \\
\hline Training & 1.000 & 0.998 & 1.000 & 1.000 \\
\hline Validation & 0.997 & 0.986 & 0.994 & 0.964 \\
\hline Testing & 0.965 & 0.950 & 0.870 & 0.938 \\
\hline \multicolumn{5}{|c|}{$\begin{array}{l}\text { B. Performance of the } \\
\text { models for postoperative }\end{array}$} \\
\hline \multicolumn{5}{|l|}{ MDFN } \\
\hline Training & 0.928 & 0.855 & 0.897 & 0.808 \\
\hline Validation & 0.881 & 0.826 & 0.746 & 0.912 \\
\hline Testing & 0.904 & 0.825 & 0.977 & 0.766 \\
\hline \multicolumn{5}{|l|}{ VGG } \\
\hline Training & 0.953 & 0.901 & 0.855 & 0.922 \\
\hline Validation & 0.805 & 0.778 & 0.887 & 0.581 \\
\hline Testing & 0.804 & 0.758 & 0.872 & 0.656 \\
\hline \multicolumn{5}{|l|}{ FCN } \\
\hline Training & 0.807 & 0.789 & 0.759 & 0.723 \\
\hline Validation & 0.776 & 0.791 & 0.550 & 0.936 \\
\hline Testing & 0.797 & 0.813 & 0.652 & 0.829 \\
\hline $\begin{array}{l}\text { C. Performance of the } \\
\text { models for postoperative } \\
\text { IMH status prediction }\end{array}$ & AUC & $\mathrm{ACC}$ & SPE & SEN \\
\hline \multicolumn{5}{|l|}{ MDFN } \\
\hline Training & 0.999 & 0.988 & 0.989 & 0.987 \\
\hline Validation & 0.974 & 0.901 & 1.000 & 0.865 \\
\hline Testing & 0.947 & 0.875 & 0.815 & 0.979 \\
\hline \multicolumn{5}{|l|}{ VGG } \\
\hline Training & 0.969 & 0.901 & 0.955 & 0.880 \\
\hline Validation & 0.891 & 0.840 & 0.782 & 0.873 \\
\hline Testing & 0.836 & 0.755 & 0.800 & 0.762 \\
\hline \multicolumn{5}{|l|}{ FCN } \\
\hline Training & 0.873 & 0.782 & 0.733 & 0.876 \\
\hline Validation & 0.926 & 0.828 & 0.800 & 0.954 \\
\hline Testing & 0.768 & 0.717 & 0.625 & 0.892 \\
\hline
\end{tabular}

ACC, accuracy; AUC, the area under the receiver operating characteristic curve; FCN, fully connected network; IMH, idiopathic macular hole; MDFN, multimodal deep fusion network; MH, macular hole; SEN, sensitivity; SPE, specificity; VGG, Visual Geometry Group.

of 0.904 , with an accuracy of 0.825 , specificity of 0.977 and sensitivity of 0.766 (table $2 \mathrm{~B}$ and figure $3 \mathrm{~B}$ ). For IMH status prediction, the AUC of the MDFN model was 0.999 in the training set, with an accuracy of 0.988 , specificity of 0.989 and sensitivity of 0.987 . In the validation set, the AUC of the MDFN model was 0.974 , with an accuracy of 0.901 , specificity of 1.000 and sensitivity of 0.865 . For external testing, the AUC, accuracy, specificity and sensitivity of the MDFN model were 0.947 , $0.875,0.815$ and 0.979 , respectively (table $2 \mathrm{C}$ and figure $3 \mathrm{C}$ ). These results indicate that our MDFN models can provide accurate prediction of postoperative $\mathrm{MH} / \mathrm{IMH}$ status, and the accuracy of the IMH status prediction was slightly higher than that of the MH status prediction.

Additionally, the predictive performance of two unimodal DL models was also evaluated in the external testing set. For $\mathrm{MH}$ status prediction, the AUC of VGG network was 0.804 , with 0.758 accuracy, 0.872 specificity and 0.656 sensitivity. The AUC of FC network was 0.797 , with 0.813 accuracy, 0.652 specificity and 0.829 sensitivity (table $2 \mathrm{~B}$ and figure $3 \mathrm{D}, \mathrm{E}$ ). For IMH status prediction, the AUC of VGG network was 0.836 , with an accuracy of 0.755 , specificity of 0.800 and sensitivity of 0.762 . The AUC, accuracy, specificity and sensitivity of FC network were $0.768,0.717,0.625$ and 0.892 , respectively (table $2 \mathrm{C}$ and figure $3 \mathrm{~F}, \mathrm{G}$ ). These results suggest that performance of the MDFN model was significantly better than those of the unimodal DL models for $\mathrm{MH} / \mathrm{IMH}$ status prediction.

Heatmaps vividly illustrate the regions most important for decision-making process of the MDFN model (figure 4). In these heatmaps, the gap between the edges of the neuro-retina was identified as the most critical pathological region for prediction of the MH status following VILMP.

\section{DISCUSSION}

In this study, we developed a DL model that could accurately distinguish IMH from SMH secondary to high myopia or trauma based on macular OCT images. We also developed an MDFN model capable of precisely predicting the postoperative IMH status based on preoperative macular OCT images and clinical data from a multicentre dataset. Moreover, the prediction accuracy of MDFN model was better than those of unimodal prediction models, suggesting that the MDFN model could improve predictive performance by the integration of image and text features. Furthermore, the prediction accuracy of postoperative status of IMH only was better than that of all $\mathrm{MH}$. This might be due to different prognostic factors between $\mathrm{SMH}$ and $\mathrm{IMH}$, and the limited amount of SMH eyes in the study.

$\mathrm{MH}$ can be classified into two categories, IMH and SMH caused by known aetiologies such as high myopia and trauma. Treatments for IMH and for SMH caused by high myopia are different. While standard VILMP and gas tamponade is often applied to IMH, SMH due to high myopia usually needs more complicated surgical modalities, such as inverted ILM flap and silicone oil tamponade. ${ }^{14}$ Macular OCT images of SMH caused by high myopia are also different from those of the IMH, due to the deformation of the posterior ocular surface. ${ }^{12}$ Thus, it is possible to distinguish these two types of $\mathrm{MH}$ based on macular OCT images only. In the present study, we further extended the possibility to automatic classification of IMH and SMH. Despite the limited amount of the macular OCT images of SMH, our DL model still achieved an accuracy of 0.965 and an AUC of 0.950 in external testing. This might be resulted from the characteristic features of macular OCT images in eyes with SMH caused by high myopia.

Since IMH is the most common type of $\mathrm{MH}$, it is clinically important to predict the anatomical outcomes of IMH surgeries. Accurate prediction of the postoperative IMH status can alleviate patients' anxieties and help ophthalmologists make better surgical plans. In patients likely to have an unfavourable prognosis after VILMP, more advanced surgical techniques, such as inverted ILM flap and autologous ILM transplantation, can be recommended to the patients. ${ }^{10} 25$ OCT imaging is useful in measuring different aspects of IMH morphology with good repeatability and reproduction. ${ }^{19}$ Some of the OCT parameters have been used as the prognostic factors of anatomical outcomes after surgery. ${ }^{17}$ However, these OCT parameters were evaluated individually in previous studies. ${ }^{15}$ The accuracy and universality of these unifactor prediction algorithms are limited, as they only analyse the predictive ability of a single parameter, while the anatomical outcomes of IMH surgery are affected by multiple factors. ${ }^{4}{ }^{19}$ In this study, we propose an MDFN model that 

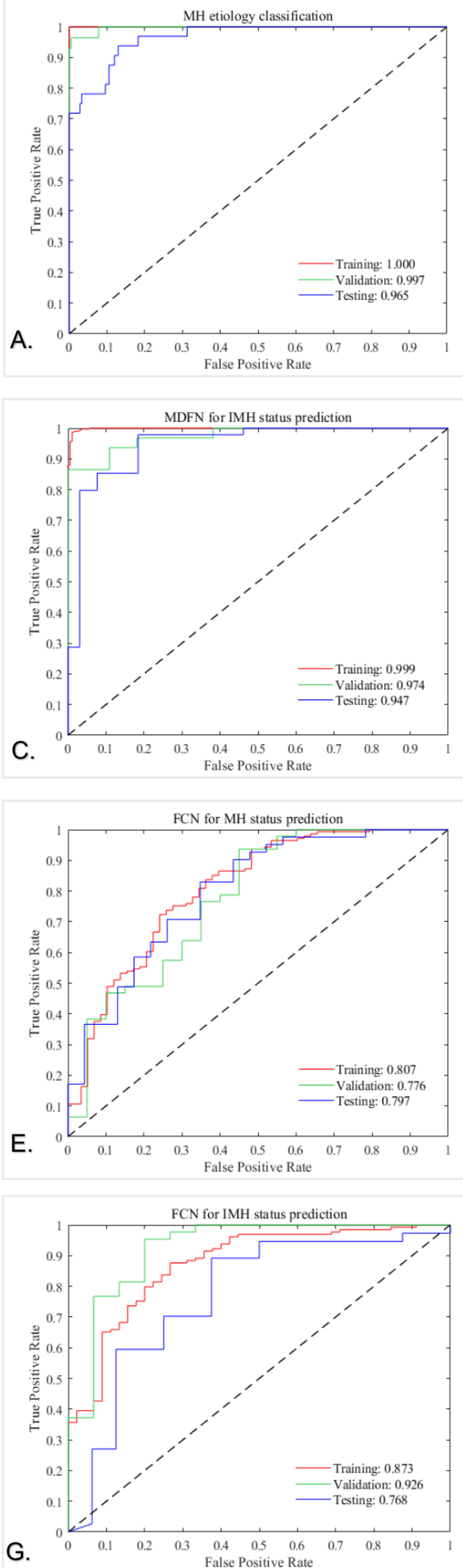
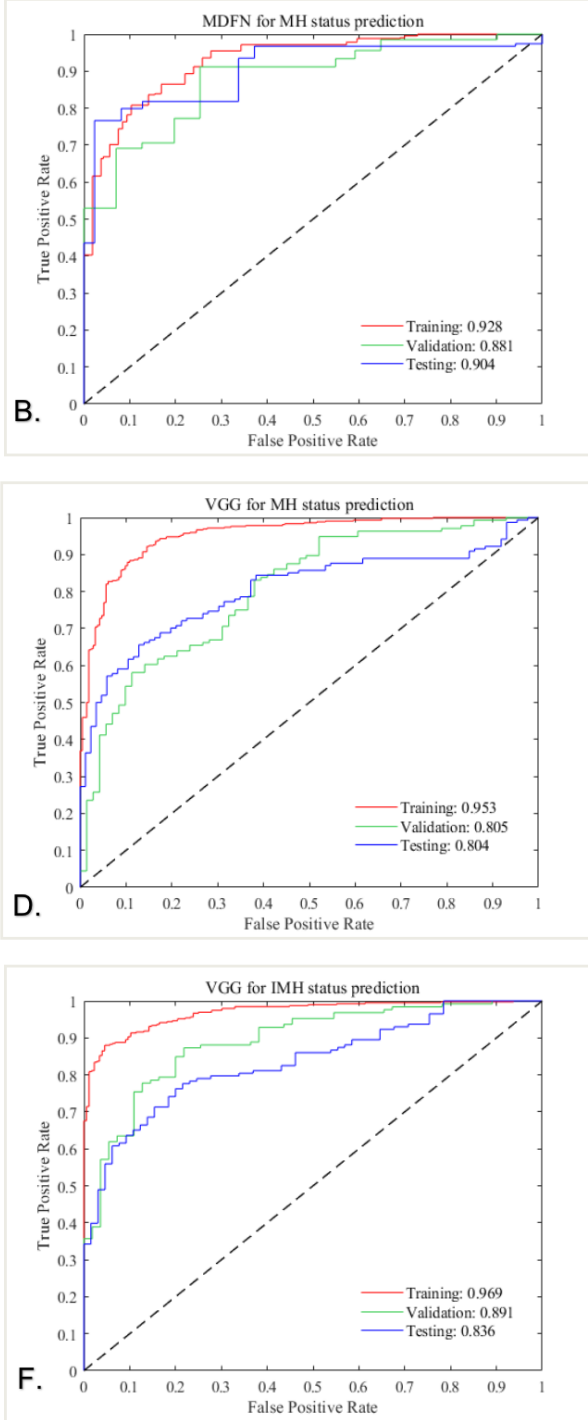

Figure 3 The receiver operating characteristic (ROC) curves of the deep learning models. (A) The ROC curves of the DL model for MH aetiology classification. (B) The ROC curves of the MDFN model for MH status prediction. (C) The ROC curves of the MDFN model for IMH status prediction. (D) The ROC curves of the VGG model for MH status prediction. (E) The ROC curves of the FCN model for MH status prediction. (F) The ROC curves of the VGG model for IMH status prediction. (G) The ROC curves of the FCN model for IMH status prediction. FCN, fully connected network; MDFN, multimodal deep fusion network; MH, macular hole; IMH, idiopathic macular hole; VGG, Visual Geometry Group.

integrates OCT image features and various clinical data of IMH to make an accurate prediction of postoperative IMH status.

Multimodal fusion of different sets of deep features has been proposed in previous studies. For instance, a multimodal DL model was developed for diagnosis of age-related macular degeneration (AMD). Two sets of deep features were extracted from colour fundus photographs and OCT images. After integrating the two types of features, the DL model exhibited an AUC of $0.969 .^{26}$ In another study, the traditional handcrafted features and deep features of colour fundus photographs were integrated to detect hard exudates for diabetic retinopathy screening, with an AUC of 0.9323 and 0.9644 in two benchmark databases, respectively. ${ }^{27}$ As mentioned before, the IMH status after surgery is affected by various factors, such as the preoperative 


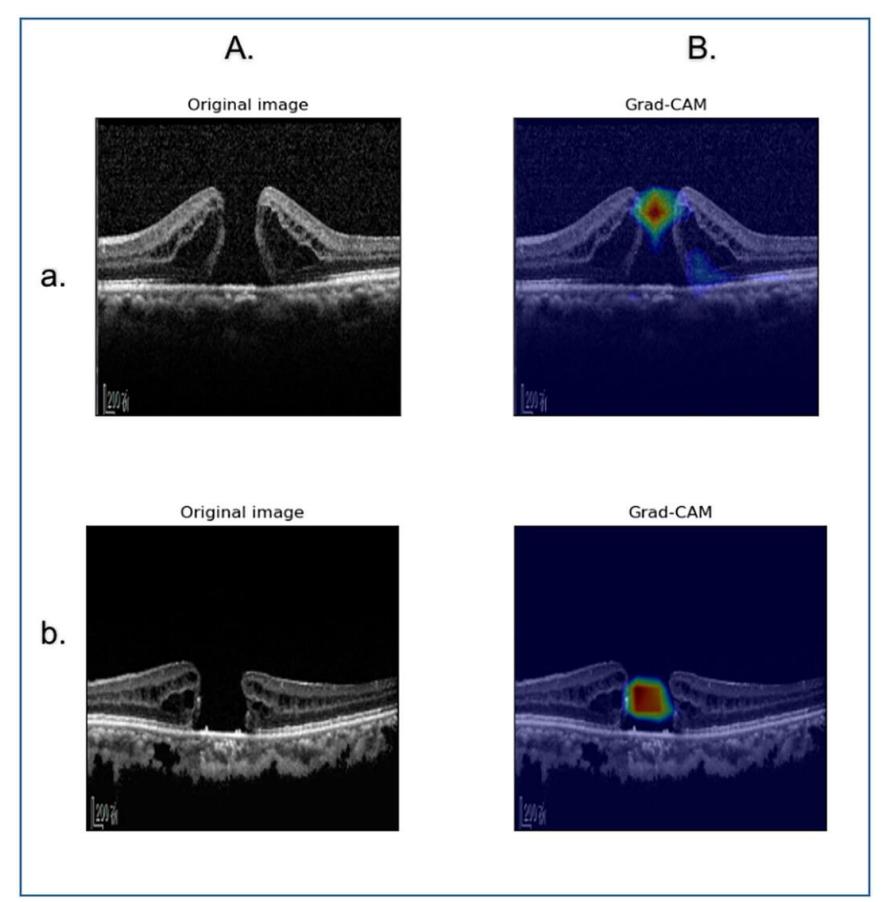

Figure 4 Visualisation heatmaps for prediction of the macular hole status after surgery. The heatmaps were generated by Gradientweighted Class Activation Mapping (Grad-CAM). The heatmaps highlight the pathological area most important for accurate prediction of the macular hole status after surgery in optical coherence tomography images.

OCT morphology and other clinical data. For the information of OCT morphology, some could be virtually measured, while the others could be embedded in the OCT image and cannot be directly measured. It is important to integrate this information to obtain an accurate prediction of IMH status after surgery. Our MDFN model contains two modules, feature extraction and feature fusion. Two types of IMH deep features were extracted, including features of the preoperative macular OCT images extracted by VGG network and features of the clinical data extracted by FC network. These two sets of features were fused by the MDFN model to predict MH status after VILMP. The better prediction accuracy of the MDFN model than those of the unimodal DL models in our study indicated that DL models based on the fusion of multimodal feature sets have better prediction performance than DL models based on unimodal feature set. Consequently, the multimodal DL models are more suitable for prediction tasks with influencing factors from multiple feature sets. Since the prognosis of many ocular diseases such as IMH and AMD is affected by factors from different feature sets (eg, image and text), it is worth applying the multimodal DL models in further studies about prognosis prediction of these ocular diseases.

Multimodal fusion refers to the combination of multiple data sets in various forms (eg, image and text) to perform target prediction, which can exploit comprehensive information provided by multimodal data. Multimodal fusion typically occurs at the feature level. The advantage of feature-level fusion lies in two aspects. First, it can obtain the most discriminatory information from original feature sets. Second, it can eliminate the redundant information resulting from the correlation between different feature sets and make real-time decisions possible. In other words, feature fusion is capable of deriving and gaining the powerful and comprehensive features important for final prediction. $^{2328}$

\section{Limitations}

There are several limitations to this study, one of which is the relatively small sample size. However, the IMH eyes included in the study were from multiple ophthalmic centres, and the promising performance of the MDFN model was validated by an independent external dataset, suggesting that our MDFN model has excellent adaptability and generalisability. Another limitation is the manual measurements of preoperative macular OCT parameters, which are subject to measurement errors. Nevertheless, the repeatability and reproducibility of manual measurements on SD-OCT have been proved good in previous studies. ${ }^{29} 30$ Recently, a fully automated 3D OCT image analysis of DL model has been developed for accurate measurement of $\mathrm{MH}$ parameters, which is potentially useful for automating $\mathrm{MH}$ measures in our DL models in the future. ${ }^{31}$ Besides, this is a preliminary study to evaluate the possibility of predicting the postoperative IMH status using an MDFN model. Prospective multiple centre trials are needed to verify the accuracy of our MDFN model. Lastly, only a small number of eyes with SMH were included in the study, and we did not include eyes with SMH secondary to macular oedema or vitrectomy, as these patients were rarely seen in clinics. Further studies are needed to predict the postoperative status of $\mathrm{SMH}$.

\section{CONCLUSIONS}

In conclusion, our DL-based models are highly accurate in classification of $\mathrm{MH}$ aetiology and prediction of postoperative $\mathrm{MH} / \mathrm{IMH}$ status. The DL-based models are potentially useful to help make automatic diagnosis and better surgical planning for patients with $\mathrm{MH}$.

\section{Author affiliations}

${ }^{1}$ Guangdong Eye Institute, Department of Ophthalmology, Guangdong Provincial People's Hospital, Guangdong Academy of Medical Sciences, Guangzhou, China ${ }^{2}$ Second School of Clinical Medicine, Southern Medical University, Guangzhou, China ${ }^{3}$ Aier Institute of Refractive Surgery, Refractive Surgery Center, Guangzhou Aier Eye Hospital, Guangzhou, China

${ }^{4}$ Aier School of Ophthalmology, Central South University, Changsha, China ${ }^{5}$ School of Computer Science and Engineering, South China University of Technology, Guangzhou, China

${ }^{6}$ State Key Laboratory of Ophthalmology, Zhongshan Ophthalmic CenterSun, Yat-sen University, Guangzhou, China

${ }^{7}$ Department of Opthalmology, the First Affiliated Hospital of Kunming Medical University, Kunming, China

${ }^{8}$ Department of Ophthalmology, Zhujiang Hospital of Southern Medical University, Guangzhou, China

${ }^{9}$ Center of Precision Medicine, Sun Yat-sen University, Guangzhou, China

Acknowledgements The authors thank the School of Computer Science and Engineering, South China University of Technology for their technical assistance with the DL system; the Zhongshan Ophthalmic Centre, the Department of Ophthalmology in the Zhujiang Hospital of Southern Medical University and the Department of Ophthalmology in the First Affiliated Hospital of Kunming Medical University for contributing OCT images and electronic medical records for training, validating and testing DL-based models.

Contributors $\mathrm{HY}, \mathrm{HL}$ and TL had full access to all the data in the study and takes responsibility for the integrity of the data and the accuracy of the data analysis. Conception and design: HL, HY, TL, HC, YX, YH and WQ. Acquisition, analysis or interpretation of data: YX, YF, YW, Yu H, SF, X Zeng, LY, WQ, BZ, QW, BL and ZL. Drafting of the manuscript: $Y X, Y H$ and WQ. Critical revision of the manuscript for important intellectual content: HY, HL, HC, YY, XW, WL and X Zhang. Statistical analysis: $Y X$ and WQ. Obtained funding: $\mathrm{HL}, \mathrm{HY}$ and $\mathrm{YH}$. Administrative, technical or material support: all authors. Supervision: HY, HL and TL.

Funding This study was supported by the Science and Technology Planning Projects of Guangdong Province (2018B010109008 to HL), National Natural Science Foundation of China (81870663 to HY; 82070972 to TL), the Science and 
Technology Programme of Guangzhou (202002030074 to HY), the Outstanding Young Talent Trainee Programme of Guangdong Provincial People's Hospital (KJ012019087 to HY), the GDPH Scientific Research Funds for Leading Medical Talents and Distinguished Young Scholars in Guangdong Province (KJ012019457 to HY), the talent introduction fund of Guangdong Provincial People's Hospital (Y012018145 to HY), the Technology Innovation Guidance Program of Hunan Province (2018SK50106 to YH), the Science Research Foundation of Aier Eye Hospital Group (AM1909D2 to YH), Guangzhou Key Laboratory Project (20200201006 to HL).

Disclaimer The funders had no role in the design and conduct of the study; collection, management, analysis and interpretation of the data; preparation, review or approval of the manuscript and decision to submit the manuscript for publication.

Competing interests None declared.

Patient consent for publication Not required.

Ethics approval The study was conducted according to the Declaration of Helsinki and was approved by the Institutional Review Board of Guangdong Provincial People's Hospital (GPPH, No. GDREC2020067H). Informed consent was taken from all patients.

Provenance and peer review Not commissioned; externally peer reviewed.

Data availability statement Data are available on reasonable request (e-mail: yuhonghua@gdph.org.cn).

Supplemental material This content has been supplied by the author(s). It has not been vetted by BMJ Publishing Group Limited (BMJ) and may not have been peer-reviewed. Any opinions or recommendations discussed are solely those of the author(s) and are not endorsed by BMJ. BMJ disclaims all liability and responsibility arising from any reliance placed on the content. Where the content includes any translated material, BMJ does not warrant the accuracy and reliability of the translations (including but not limited to local regulations, clinical guidelines, terminology, drug names and drug dosages), and is not responsible for any error and/or omissions arising from translation and adaptation or otherwise.

Open access This is an open access article distributed in accordance with the Creative Commons Attribution Non Commercial (CC BY-NC 4.0) license, which permits others to distribute, remix, adapt, build upon this work non-commercially, and license their derivative works on different terms, provided the original work is properly cited, appropriate credit is given, any changes made indicated, and the use is non-commercial. See: http://creativecommons.org/licenses/by-nc/4.0/.

\section{ORCID iDs}

Yu Xiao http://orcid.org/0000-0002-0911-6857

Yijun Hu http://orcid.org/0000-0002-6424-7905

Qiaowei Wu http://orcid.org/0000-0002-3054-2682

Tao Li http://orcid.org/0000-0001-8064-871X

Haotian Lin http://orcid.org/0000-0003-4672-9721

Honghua Yu http://orcid.org/0000-0002-0782-346X

\section{REFERENCES}

1 Takahashi A, Yoshida A, Nagaoka T, et al. Idiopathic full-thickness macular holes and the vitreomacular interface: a high-resolution spectral-domain optical coherence tomography study. Am J Ophthalmol 2012;154:881-92. e882.

2 McCannel CA, Ensminger JL, Diehl NN, et al. Population-based incidence of macular holes. Ophthalmology 2009;116:1366-9.

3 Thapa SS, Thapa R, Paudyal I, et al. Prevalence and pattern of vitreo-retinal diseases in Nepal: the Bhaktapur glaucoma study. BMC Ophthalmol 2013;13:9.

4 Ali FS, Stein JD, Blachley TS, et al. Incidence of and risk factors for developing idiopathic macular hole among a diverse group of patients throughout the United States. JAMA Ophthalmol 2017;135:299-305.

5 Duker JS, Kaiser PK, Binder S, Susanne B, et al. The International Vitreomacular traction Study Group classification of vitreomacular adhesion, traction, and macular hole. Ophthalmology 2013;120:2611-9.
6 Chew EY, Sperduto RD, Hiller R, et al. Clinical course of macular holes: the eye disease case-control study. Arch Ophthalmol 1999;117:242-6.

7 Gross JG, Gross Jeffrey G. Late reopening and spontaneous closure of previously repaired macular holes. Am J Ophthalmol 2005:140:556-8.

8 Yao Y, Qu J, Dong C, et al. The impact of extent of internal limiting membrane peeling on anatomical outcomes of macular hole surgery: results of a 54-week randomized clinical trial. Acta Ophthalmol 2019;97:303-12.

9 Abbey AM, Van Laere L, Shah AR, et al. Recurrent macular holes in the era of small-gauge vitrectomy: a review of incidence, risk factors, and outcomes. Retina 2017:37:921-4.

10 Michalewska Z, Michalewski J, Adelman RA, et al. Inverted internal limiting membrane flap technique for large macular holes. Ophthalmology 2010;117:2018-25.

11 Valldeperas X, Wong D. Is it worth reoperating on macular holes? Ophthalmology 2008;115:158-63.

12 Lin C-W, Ho T-C, Yang C-M. The development and evolution of full thickness macular hole in highly myopic eyes. Eye 2015;29:388-96.

13 Tang YF, Chang A, Campbell WG, et al. Surgical management of traumatic macular hole: optical coherence tomography features and outcomes. Retina 2020;40:290-8.

14 Wu T-T, Kung Y-H, Chang C-Y, et al. Surgical outcomes in eyes with extremely high myopia for macular hole without retinal detachment. Retina 2018;38:2051-5.

15 Wakely L, Rahman R, Stephenson J. A comparison of several methods of macular hole measurement using optical coherence tomography, and their value in predicting anatomical and visual outcomes. Br J Ophthalmol 2012;96:1003-7.

16 Ip MS, Baker BJ, Duker JS, et al. Anatomical outcomes of surgery for idiopathic macular hole as determined by optical coherence tomography. Arch Ophthalmol 2002:120:29-35.

17 Ullrich S, Haritoglou C, Gass C, et al. Macular hole size as a prognostic factor in macular hole surgery. Br J Ophthalmol 2002:86:390-3.

18 Kusuhara S, Teraoka Escaño MF, Fujii S, et al. Prediction of postoperative visual outcome based on hole configuration by optical coherence tomography in eyes with idiopathic macular holes. Am J Ophthalmol 2004;138:709-16.

19 Ruiz-Moreno JM, Staicu C, Piñero DP, et al. Optical coherence tomography predictive factors for macular hole surgery outcome. Br J Ophthalmol 2008;92:640-4.

20 Kermany DS, Goldbaum M, Cai W, et al. Identifying medical diagnoses and treatable diseases by image-based deep learning. Cell 2018:172:e1129:1122-31.

21 Liu H, Li L, Wormstone IM, et al. Development and validation of a deep learning system to detect glaucomatous optic neuropathy using fundus Photographs. JAMA Ophthalmol 2019;137:1353.

22 Nagasawa T, Tabuchi H, Masumoto H, et al. Accuracy of deep learning, a machine learning technology, using ultra-wide-field fundus ophthalmoscopy for detecting idiopathic macular holes. PeerJ 2018;6:e5696.

23 Jingdong W, Zhen W, Ting Z. Deeply-fused nets. ar Xiv 2016.

24 Rawat W, Wang Z, Waseem R. Deep convolutional neural networks for image classification: a comprehensive review. Neural Comput 2017:29:2352-449.

25 Morizane Y, Shiraga F, Kimura S, et al. Autologous transplantation of the internal limiting membrane for refractory macular holes. Am J Ophthalmol 2014;157:861-9. e861.

26 Yoo TK, Choi JY, Seo JG, et al. The possibility of the combination of OCT and fundus images for improving the diagnostic accuracy of deep learning for agerelated macular degeneration: a preliminary experiment. Med Biol Eng Comput 2019:57:677-87.

27 Wang H, Yuan G, Zhao X, et al. Hard exudate detection based on deep model learned information and multi-feature joint representation for diabetic retinopathy screening. Comput Methods Programs Biomed 2020;191:105398.

28 Ke L, Changqing Z, Shuhui B. Multi-modal feature fusion for geographic image annotation. Pattern Recognit 2018;73:1-14.

29 Rahman W, Chen FK, Yeoh J, et al. Repeatability of manual subfoveal choroida thickness measurements in healthy subjects using the technique of enhanced depth imaging optical coherence tomography. Invest Ophthalmol Vis Sci 2011:52:2267-71.

30 Chhablani J, Barteselli $\mathrm{G}$, Wang $\mathrm{H}$, et al. Repeatability and reproducibility of manual choroidal volume measurements using enhanced depth imaging optical coherence tomography. Invest Ophthalmol Vis Sci 2012;53:2274-80.

31 Murphy DC, Nasrulloh AV, Lendrem C, et al. Predicting postoperative vision for macular hole with automated image analysis. Ophthalmol Retina 2020;4:1211-3. 